\title{
Aircraft Engine On-Line Diagnostics Through Dual-Channel Sensor Measurements: Development of a Baseline System
}

Takahisa Kobayashi

ASRC Aerospace Corporation, Cleveland, Ohio

Donald L. Simon

Glenn Research Center, Cleveland, Ohio 


\section{NASA STI Program . . . in Profile}

Since its founding, NASA has been dedicated to the advancement of aeronautics and space science. The NASA Scientific and Technical Information (STI) program plays a key part in helping NASA maintain this important role.

The NASA STI Program operates under the auspices of the Agency Chief Information Officer. It collects, organizes, provides for archiving, and disseminates NASA's STI. The NASA STI program provides access to the NASA Aeronautics and Space Database and its public interface, the NASA Technical Reports Server, thus providing one of the largest collections of aeronautical and space science STI in the world. Results are published in both non-NASA channels and by NASA in the NASA STI Report Series, which includes the following report types:

- TECHNICAL PUBLICATION. Reports of completed research or a major significant phase of research that present the results of NASA programs and include extensive data or theoretical analysis. Includes compilations of significant scientific and technical data and information deemed to be of continuing reference value. NASA counterpart of peer-reviewed formal professional papers but has less stringent limitations on manuscript length and extent of graphic presentations.

- TECHNICAL MEMORANDUM. Scientific and technical findings that are preliminary or of specialized interest, e.g., quick release reports, working papers, and bibliographies that contain minimal annotation. Does not contain extensive analysis.

- CONTRACTOR REPORT. Scientific and technical findings by NASA-sponsored contractors and grantees.

- CONFERENCE PUBLICATION. Collected papers from scientific and technical conferences, symposia, seminars, or other meetings sponsored or cosponsored by NASA.

- SPECIAL PUBLICATION. Scientific, technical, or historical information from NASA programs, projects, and missions, often concerned with subjects having substantial public interest.

- TECHNICAL TRANSLATION. Englishlanguage translations of foreign scientific and technical material pertinent to NASA's mission.

Specialized services also include creating custom thesauri, building customized databases, organizing and publishing research results.

For more information about the NASA STI program, see the following:

- Access the NASA STI program home page at http://www.sti.nasa.gov

- E-mail your question via the Internet to help@ sti.nasa.gov

- Fax your question to the NASA STI Help Desk at 301-621-0134

- Telephone the NASA STI Help Desk at 301-621-0390

- Write to: NASA Center for AeroSpace Information (CASI) 7115 Standard Drive Hanover, MD 21076-1320 
NASA/TM-2008-215228

\section{Aircraft Engine On-Line Diagnostics Through Dual-Channel Sensor Measurements: Development of a Baseline System}

Takahisa Kobayashi

ASRC Aerospace Corporation, Cleveland, Ohio

Donald L. Simon

Glenn Research Center, Cleveland, Ohio

Prepared for the

Turbo Expo 2008 Gas Turbine Technical Congress and Exposition sponsored by the American Society of Mechanical Engineers Berlin, Germany, June 9-13, 2008

National Aeronautics and

Space Administration

Glenn Research Center

Cleveland, Ohio 44135 


\section{Acknowledgments}

This research was funded by the NASA Aviation Safety Program as a task under the Integrated Vehicle Health Management Project.

This report is a preprint of a paper intended for presentation at a conference.

Because changes may be made before formal publication, this preprint is made available with the understanding that it will not be cited or reproduced without the permission of the author.

Level of Review: This material has been technically reviewed by technical management.

Available from

NASA Center for Aerospace Information 7115 Standard Drive

Hanover, MD 21076-1320
National Technical Information Service 5285 Port Royal Road Springfield, VA 22161

Available electronically at http://gltrs.grc.nasa.gov 


\title{
Aircraft Engine On-Line Diagnostics Through Dual-Channel Sensor Measurements: Development of a Baseline System
}

\author{
Takahisa Kobayashi \\ ASRC Aerospace Corporation \\ Cleveland, Ohio 44135 \\ Donald L. Simon \\ National Aeronautics and Space Administration \\ Glenn Research Center \\ Cleveland, Ohio 44135
}

\begin{abstract}
In this paper, a baseline system which utilizes dual-channel sensor measurements for aircraft engine on-line diagnostics is developed. This system is composed of a linear on-board engine model (LOBEM) and fault detection and isolation (FDI) logic. The LOBEM provides the analytical third channel against which the dual-channel measurements are compared. When the discrepancy among the triplex channels exceeds a tolerance level, the FDI logic determines the cause of the discrepancy. Through this approach, the baseline system achieves the following objectives: 1) anomaly detection, 2) component fault detection, and 3) sensor fault detection and isolation. The performance of the baseline system is evaluated in a simulation environment using faults in sensors and components.
\end{abstract}

\section{INTRODUCTION}

Aircraft gas turbine engines have evolved into a highly complex and sophisticated system to meet ever-increasing demands. The evolution of aircraft engine technologies has been driven by the need for improved fuel efficiency, reliability, operability, availability, and maintainability [1,2]. The reliability aspect of modern aircraft engines has been enhanced by equipping the engines with a dual channel fullauthority digital electronic control (FADEC). In this setup, a single engine parameter is measured by a dual-channel sensor, ${ }^{\dagger}$ and the FADEC receives redundant measurements through dual channels. If a single channel fails, this failure is accommodated by utilizing the measurement on the other channel.
Such an accommodation action can be taken only if the identity of the faulty sensor and its failed channel are known. Thus, in order to fully utilize the available redundancy for sensor fault accommodation, the ability to diagnose the sensors on line (real-time, in-flight) is required. The sensor fault detection and isolation process is initiated by cross-checking the redundant measurements of each dual-channel sensor. If both channels agree within a pre-established tolerance, the measurements on both channels are acceptable. If not, the cross-check fails, and one of the dual channels is considered faulty. A challenge arises in the subsequent process of identifying the faulty channel. Even if redundant measurements do not agree with each other, both of them may pass the range and rate checks [3]. Such a failure is called an in-range sensor fault and causes some difficulty in determining which channel is the failed one.

The above problem can be addressed by providing an analytical third channel. This third channel functions as a referee in the decision making; the channel that disagrees most with the referee is likely the faulty channel. The analytical third channel is embedded within the FADEC in the form of an analytical representation of the real engine. This analytical representation is called an on-board engine model (OBEM) and has been a key element in on-line fault detection and

\footnotetext{
${ }^{\dagger}$ A dual-channel sensor is defined in this paper as a device which produces two measurements of the same engine parameter. The redundant measurements are referred to as channel A and channel B measurements. It is assumed that failures can occur in either one or both channels of the sensor.
} 
accommodation since the advent of the FADEC [4,5]. The OBEM captures the real engine's nominal behavior to some extent and provides expected engine output values in real time. In Ref. [3], a linear on-board engine model (LOBEM), which is composed of piecewise linear models generated at multiple operating conditions, is utilized for on-line sensor fault diagnostics.

In this paper, an on-line diagnostic system that utilizes the LOBEM as an analytical third channel is developed for the aircraft engine application. This system is referred to as the baseline system, and it has a simple structure composed of the LOBEM and fault detection and isolation (FDI) logic. When the discrepancy among the triplex channels exceeds a tolerance level, the FDI logic determines the cause of the discrepancy. Through this approach, the baseline system achieves the following objectives: 1) anomaly detection, 2) component fault detection, and 3) sensor fault detection and isolation. Because of its simplicity, the baseline system can be executed with the computing power currently available in practice. Therefore, diagnostic performance representative of what is potentially achievable in the field can be determined through evaluation of the baseline system.

In the following section, two main components of the baseline system-the LOBEM and the FDI logic-are discussed in detail. The influence of engine health degradation on the on-line diagnostic performance is also discussed. Then, the design approach is applied to a large commercial aircraft engine model. The performance of the baseline system is evaluated using simulated faults in sensors and components.

$\begin{array}{ll}\text { NOMENCLATURE } \\ \text { BST } & \text { Booster } \\ \text { CFS } & \text { Component fault signature } \\ \text { FADEC } & \text { Full Authority Digital Electronic Control } \\ \text { FDI } & \text { Fault Detection and Isolation } \\ \text { HPC } & \text { High Pressure Compressor } \\ \text { HPT } & \text { High Pressure Turbine } \\ \text { LOBEM } & \text { Linear On-Board Engine Model } \\ \text { LPT } & \text { Low Pressure Turbine } \\ \text { P2 } & \text { Engine inlet pressure } \\ \text { P25 } & \text { HPC inlet pressure } \\ \text { Pamb } & \text { Ambient pressure } \\ \text { PLA } & \text { Power Lever Angle } \\ \text { PS3 } & \text { Combustor inlet static pressure } \\ \text { T2 } & \text { Engine inlet temperature } \\ \text { T25 } & \text { HPC inlet temperature } \\ \text { T3 } & \text { Combustor inlet temperature } \\ \text { T49 } & \text { LPT inlet temperature } \\ \text { TMHS23 } & \text { BST metal temperature } \\ \text { TMHS3 } & \text { HPC metal temperature } \\ \text { TMHS41 } & \text { HPT nozzle metal temperature } \\ \text { TMHS42 } & \text { HPT metal temperature } \\ \text { TMHS5 } & \text { LPT metal temperature } \\ \text { TMHSBC } & \text { Combustor case metal temperature } \\ & \end{array}$

TMHSBL Combustor liner metal temperature

VBV Variable bleed valve

VSV Variable stator vane

WF36 Fuel flow

XN12 Fan speed, measured

XN25 Core speed, measured

$\mathrm{XNH} \quad$ Core speed, actual

XNL Fan speed, actual

$e \quad$ Environmental parameter vector

$h \quad$ Health parameter vector

$h_{\text {ref }} \quad$ Reference health condition vector

$u_{c m d} \quad$ Control command vector

$v \quad$ Sensor noise vector

$x \quad$ State variable vector

$y \quad$ Sensor output vector (controls/diagnostics)

z Sensor output vector (ambient/engine inlet)

\section{DEVELOPMENT OF THE BASELINE SYSTEM FOR ON-LINE DIAGNOSTICS}

The objective of on-line diagnostics for aircraft engines is to detect, and if possible isolate, any fault as early as possible. With timely detection and accurate isolation of the fault, the necessary corrective actions can be taken to avoid undesirable engine operation and maintenance costs. To achieve this objective, the baseline system continuously monitors engine outputs for anomalous signatures induced by faults. The baseline system developed in this paper is composed of the LOBEM and FDI logic. Each component is described in this section.

\section{Linear On-Board Engine Model (LOBEM)}

An aircraft engine under consideration for on-line diagnostics is described by nonlinear equations of the following form:

$$
\begin{aligned}
& \dot{x}=f\left(x, h, u_{c m d}, e\right) \\
& y=g\left(x, h, u_{c m d}, e\right)
\end{aligned}
$$

The vectors $x, u_{c m d}$, and $e$ contain state variables, control command inputs, and environmental parameters, respectively. The vector $h$ contains health parameters that indicate the engine health condition. Health parameters are efficiencies and flow capacities of the engine components such as the compressors and turbines. As they deviate from their nominal health condition, the performance delivered by each component degrades. For given inputs, the nonlinear functions $f$ and $g$ generate the state derivative vector $\dot{x}$ and engine output vector $y$. The engine outputs are measured by sensors with dualchannels A and B as follows:

$$
\begin{aligned}
& y_{\mathrm{A}}=y+v_{\mathrm{A}} \\
& y_{\mathrm{B}}=y+v_{\mathrm{B}}
\end{aligned}
$$


The vectors $v_{\mathrm{A}}$ and $v_{\mathrm{B}}$ represent the zero-mean, normally distributed white noise that corrupts the measurements on dual channels.

The baseline system described in this paper uses the LOBEM as an analytical third channel. The LOBEM is developed through the linearization of a high-fidelity nonlinear engine model in the following form:

$$
\begin{aligned}
& \dot{\bar{x}}=\bar{f}\left(\bar{x}, h_{r e f}, u_{c m d}, e\right) \\
& \bar{y}=\bar{g}\left(\bar{x}, h_{r e f}, u_{c m d}, e\right)
\end{aligned}
$$

The vectors $\bar{x}$ and $\bar{y}$ contain the state and output variables of the nonlinear engine model, respectively. The vector $h_{r e f}$ contains health parameters that indicate the health condition of the nonlinear engine model. The health condition prescribed by the vector $h_{r e f}$ is called the "health baseline" since it is the reference health condition at which the nonlinear model is linearized. It should be noted that the form of the nonlinear engine model is similar to the assumed form of the actual engine in Eq. (1). It is critical in any model-based analysis to have a model that accurately represents the actual system.

Through the linearization of the nonlinear engine model in Eq. (3), the LOBEM is developed. The LOBEM is represented by the linear state-space equations of the following form:

$$
\begin{aligned}
& \dot{x}_{\text {LOBEM }}=A\left(x_{\text {LOBEM }}-x_{s S}\right)+B\left(u_{c m d}-u_{s S}\right) \\
& y_{\text {LOBEM }}=C\left(x_{\text {LOBEM }}-x_{s S}\right)+D\left(u_{c m d}-u_{s s}\right)+y_{s s}
\end{aligned}
$$

The vectors $x_{\text {LOBEM }}$ and $y_{\text {LOBEM }}$ contain the state and output variables of the LOBEM, respectively. The state-space matrices $A, B, C$, and $D$ are of appropriate dimensions. The vectors $x_{s s}, y_{s s}$, and $u_{s s}$ contain the steady-state values at which the nonlinear engine model is trimmed for linearization. A set of state-space matrices and steady-state vectors represents a linear engine model at a specific operating condition. To cover a wide operational range of the aircraft engine, linear models must be generated at multiple operating conditions. Such linear models generated at multiple operating conditions are called piecewise linear models and implemented on the FADEC in table lookup form. For real-time execution of Eq. (4), the piecewise linear models are interpolated as the operating condition of the actual engine moves from one point to another. Because of its simple structure, the LOBEM in Eq. (4) is suited for on-line application.

\section{Fault Detection and Isolation (FDI) Logic}

Another component of the baseline system is the FDI logic. The FDI logic compares the triplex channels $\left(y_{\mathrm{A}}, y_{\mathrm{B}}\right.$, and $y_{\text {LOBEM }}$ ) and determines a root cause when an anomalous signature is detected in these channels.

There are multiple comparisons that are carried out by the FDI logic. The first comparison is a cross-check between channels A and B. There are a total of $m$ engine parameters which are measured by $m$ dual-channel sensors. For each measured parameter, the residual is computed as follows:

$$
r^{i}=\frac{y_{\mathrm{A}}^{i}-y_{\mathrm{B}}^{i}}{\sigma_{i}}, \quad i=1, \ldots, m
$$

where $\sigma_{i}$ indicates the standard deviation of the measurement uncertainty of the $i^{\text {th }}$ sensor. The residual in Eq. (5) is called the "dual-channel residual." The dual-channel residual for each sensor is compared against a pre-established threshold, $\tau_{D R}^{i}$. If the dual-channel residual does not exceed the threshold, the redundant measurements on both channels are acceptable. Otherwise, at least one of the dual channels is faulty. This process can only determine whether at least one channel of the dual-channel sensor is faulty, but not which channel is faulty.

In addition to the comparison between the dual channels, the comparison of the dual channels against the model output is performed as follows:

$$
\begin{aligned}
& r_{\mathrm{A}}^{i}=\frac{y_{\mathrm{A}}^{i}-y_{\text {LOBEM }}^{i}}{\sigma_{i}} \\
& r_{\mathrm{B}}^{i}=\frac{y_{\mathrm{B}}^{i}-y_{\text {LOBEM }}^{i}}{\sigma_{i}}
\end{aligned}
$$

The residual in Eq. (6) is called the "analytical residual." The analytical residual computed for each channel of each sensor is compared against a pre-established threshold, $\tau_{A R}^{i}$. The LOBEM generates the expected output values of the engine operating without any faults. If an analytical residual exceeds a threshold, it indicates the existence of an anomaly.

Through the comparison of triplex channels, the FDI logic determines the root cause of the problem based on the following assumptions:

- $\quad$ Only one sensor may be faulty at a time, i.e., either one or both channels of this sensor may be faulty at a time.

- Multiple components may be faulty at a time.

- Only one of the above two conditions occurs at a time.

The FDI logic indicates one of the following conditions: 1) sensor fault detected, 2) sensor fault isolated, 3) component fault detected, or 4) anomaly detected. Each of these conditions is discussed in the following section, and a flow chart of the FDL logic is given in the Appendix. 
Sensor Fault Detection. The FDI logic indicates that "a sensor fault is detected" when one of the dual-channel residuals in Eq. (5) exceeds the threshold $\tau_{D R}^{i}$, but all analytical residuals in Eq. (6) remain below the threshold $\tau_{A R}^{i}$. This scenario happens depending on the fault magnitude and the threshold values. In this condition, the identity of a faulty dual-channel sensor is determined, but the identity of its failed channel cannot be determined.

Sensor Fault Isolation. The FDI logic indicates that "a sensor fault is isolated" when the dual-channel residual of a particular sensor exceeds the threshold $\tau_{D R}^{i}$, and also, this sensor's analytical residual exceeds the threshold $\tau_{A R}^{i}$ in either one or both channels. If the threshold violation of the analytical residual occurs only in one channel, the channel that caused this violation is identified as the faulty one. If the threshold violation occurs in both channels, both channels of this particular sensor are considered faulty. Thus, the identity of a faulty sensor and the identity of its failed channel are determined.

Component Fault Detection. Although sensor faults are most likely to occur [6], aircraft engines may experience faults in rotating components. When the engine experiences a component fault, multiple engine parameters deviate from their nominal condition values. As a result, the analytical residuals in Eq. 6 will change, but the dual-channel residuals in Eq. 5 are unaffected as long as the sensors are functioning normally. Therefore, a component fault can be detected through the observation of changes in the analytical residuals. Since the analytical residuals are influenced not only by a component fault but also by a sensor fault, care must be paid to observe a feature that is intrinsic to a component fault.

A component fault generally causes an increase in the analytical residuals of multiple sensors. This is a major difference from the single sensor fault case, where the analytical residual of one sensor prominently increases. To utilize this knowledge, a component fault signature (CFS) is computed for each of the dual channels $\mathrm{A}$ and $\mathrm{B}$ as follows:

$$
\begin{aligned}
& C F S_{\mathrm{A}}=\sum_{i=1}^{m}\left(\frac{r_{\mathrm{A}}^{i}}{\tau_{A R}^{i}}\right)^{2}-\alpha \\
& C F S_{\mathrm{B}}=\sum_{i=1}^{m}\left(\frac{r_{\mathrm{B}}^{i}}{\tau_{A R}^{i}}\right)^{2}-\beta
\end{aligned}
$$

where

$$
\alpha=\max _{i=1, \ldots, m}\left\{\left(\frac{r_{\mathrm{A}}^{i}}{\tau_{A R}^{i}}\right)^{2}\right\}, \quad \beta=\max _{i=1, \ldots, m}\left\{\left(\frac{r_{\mathrm{B}}^{i}}{\tau_{A R}^{i}}\right)^{2}\right\}
$$

With a total of $m$ dual-channel sensors, $m$ analytical residuals are calculated for each channel. The scalars $\alpha$ and $\beta$ in Eq. (8) are the maximum ratio of the analytical residual to the threshold $\tau_{A R}^{i}$ for channel A and B, respectively, among the $m$ dual-channel sensors. These scalars correspond to the analytical residual of a given channel that is largest in magnitude with respect to its threshold. This maximum ratio value is subtracted from the summation in Eq. (7).

When a single sensor is faulty, the analytical residual of the faulty sensor will increase while the analytical residuals of other sensors are unaffected. Consequently, the analytical residual of the faulty sensor becomes the maximum among the $m$ analytical residuals, and this maximum is subtracted in the CFS calculation. Therefore, the CFS value is unaffected by a single sensor fault. When a component is faulty, multiple engine parameters are affected, multiple analytical residuals will increase, and consequently, the CFS value will increase. As such, the increase in the CFS value indicates the existence of a component fault.

The CFS for each channel is compared against a preestablished threshold, $\tau_{C F S}$. When the CFS exceeds the threshold in at least one channel while all dual-channel residuals remain below the threshold $\tau_{D R}^{i}$, the FDI logic indicates that "a component fault is detected."

Anomaly Detection. The FDI logic indicates that "an anomaly is detected" when it determines that something is abnormal, but it is unable to classify the anomaly into one of the three categories previously listed. This occurs when at least one of the analytical residuals exceeds the threshold $\tau_{A R}^{i}$, while all dual-channel residuals and CFS remain below their corresponding thresholds. Such a scenario can be induced by different causes. One possibility is a sensor fault: one channel of a sensor is actually faulty and thus causes a threshold violation of an analytical residual, but the fault magnitude is too small to cause the dual-channel residual of this sensor to exceed its corresponding threshold. This can happen depending on the threshold values. Another possibility is a component fault. A component fault influences the analytical residuals and CFS. Depending on the damaged component, fault severity, and the noise in the dual channels, one of the analytical residuals may exceed a threshold, while the CFS remains below its threshold. As such, the cause of the threshold violation is not obvious in this scenario. The FDI logic indicates the existence of an anomaly when the specific identity of a fault cannot be determined.

It is possible to encounter a scenario where both channels of a sensor fail in a similar manner, and the dual-channel measurements remain in good agreement. In such a case, the dual-channel residual of the failed sensor does not exceed its threshold. Thus, the FDI logic indicates the existence of an anomaly, instead of a sensor fault, when the analytical residual of this sensor exceeds its threshold in at least one channel. 


\section{INFLUENCE OF HEALTH DEGRADATION}

Up to this point, the focus of this paper has been on the detection and isolation of faults that the aircraft engines may encounter. A fault is an abnormal event that happens unexpectedly. Such an event influences the observed engine behavior to some extent, and therefore, can be detected through the comparison of triplex channels. There is, however, a major phenomenon that also influences engine behavior and causes anomalous signatures in the measurements. This phenomenon is engine health degradation. Engine health degradation is a normal aging process that all aircraft engines will experience as a result of usage. It is not considered as a fault, but its influence on the engine performance grows over time. Eventually the on-line system will lose its diagnostic effectiveness if health degradation is not accounted for in the diagnostic process.

The health condition of an engine is defined by the vector $h$ in Eq. (1) which contains health parameters. The health condition at an instant of time can be expressed as follows:

$$
h=h_{k}+\Delta h
$$

The vector $h_{k}$ represents health degradation. Since health degradation progresses gradually over the lifetime of an engine, $h_{k}$ is treated as a constant vector in the time scale at which the on-line system operates (i.e., real-time operation). The subscript $k$ indicates the time index of a much longer time scale at which the change in health degradation takes place (i.e., $k$ may increment once every few flights or few days). The vector $\Delta h$ represents a component fault that can occur abruptly at any instant of time. The on-line diagnostic system aims to detect this component fault when it happens. If there is no component fault, health degradation defines the health condition of the engine at a given instant.

As discussed earlier, the LOBEM in Eq. (4) is developed at a specific health condition (e.g., nominal health condition), and this design condition is called the health baseline. As long as this health baseline and the true health condition of the real engine are in close proximity, the LOBEM accurately represents the real engine's performance. As the engine continues to degrade, the difference between the true health condition and the health baseline increases. With such an increase of health condition mismatch, the LOBEM becomes a poor representation of the real engine and consequently corrupts the diagnostic capability of the baseline system. To address this problem, the LOBEM must be adjusted or updated periodically so that it can account for the influence of health degradation.

There are a number of possible approaches for updating the LOBEM. One approach is to modify Eq. (4) into the following form:

$$
\begin{aligned}
& \dot{x}_{\text {LOBEM }}=A\left(x_{\text {LOBEM }}-x_{\text {ss }}\right)+B\left(u_{c m d}-u_{s s}\right)+L\left(\hat{h}_{k}-h_{\text {ref }}\right) \\
& y_{\text {LOBEM }}=C\left(x_{\text {LOBEM }}-x_{\text {ss }}\right)+D\left(u_{c m d}-u_{s s}\right)+M\left(\hat{h}_{k}-h_{r e f}\right)+y_{s s}
\end{aligned}
$$

As the engine degrades over time, the difference between the true health condition and the health baseline, $h_{k}-h_{\text {ref }}$, increases. Equation (10) captures the influence of this difference by incorporating the term $\hat{h}_{k}-h_{\text {ref }}$. The vector $\hat{h}_{k}$ represents the estimate of true health degradation $h_{k}$, and it is assumed that this estimate is provided by a trend monitoring algorithm as demonstrated in Ref. [7]. As long as the estimate is reasonably accurate (i.e., $\hat{h}_{k} \approx h_{k}$ ), the influence of health degradation on engine variables is accounted for through the influence coefficient matrices $L$ and $M$.

This approach appears attractive because of its simplicity. However, it has some drawbacks. One of the drawbacks is that the relationship between health parameters and engine variables must be linear, so that the influence of health degradation can be captured through the matrices $L$ and $M$. Such a linear relationship often does not hold for aircraft engines. Another drawback is that Eq. (10) does not capture the "off-design" closed-loop effect. Regardless of health degradation, aircraft engines must maintain specific variables of interest, such as fan speed or engine pressure ratio, at the target condition. To achieve this objective, the control system adjusts its commands as the engines degrade. This closed-loop control action changes the steady-state relationship of engine variables. The new relationship becomes "off-design" with respect to the original relationship at which the LOBEM was developed. As health degradation progresses over time, the off-design relationship departs further from the original, and the steadystate vectors $x_{s s}, u_{s s}$, and $y_{s s}$ which capture the original relationship become obsolete. As such, the adjustment of the LOBEM through Eq. (10) only works if the nonlinearity and the closed-loop effect are moderate.

An alternative approach to account for the influence of health degradation, while addressing the issues of nonlinearity and closed-loop effect, is to periodically update the LOBEM to a new health baseline through the re-generation of piecewise linear models. As discussed in Ref. [7], health degradation is estimated by a trend monitoring algorithm periodically over the course of the engine's life. Using this estimate, the LOBEM can be updated to a new health baseline through the following steps. First, the health baseline of the nonlinear engine model in Eq. (3) is set to the estimated health condition as follows:

$$
h_{r e f}=\hat{h}_{k}
$$

Then, at the new health baseline, the piecewise linear models are generated from the nonlinear engine model. Re-generated 
piecewise linear models take into account the influence of health degradation on engine variables as long as the health estimate is reasonably accurate. Through this process, the LOBEM in Eq. (4) is updated to a new health baseline and is able to remain as a reasonably accurate representation of the degraded engine. This update process must be completed periodically over the lifetime of the engine so that the LOBEM can operate in the vicinity of the true health condition of the degraded engine.

The drawback of this approach is its complexity. The process of re-linearizing the nonlinear engine model may have to take place on a ground-based computer with human intervention. Furthermore, after the re-linearization process, the piecewise linear models must be uploaded to the FADEC. Such complexity raises a number of questions regarding the practicality of this approach.

Updating the LOBEM is a highly challenging problem. Unless this problem is addressed, the LOBEM developed at a specific health baseline eventually becomes an obsolete representation of a degraded engine and consequently corrupts the diagnostic capability of the baseline system. In a later section, the influence of health degradation on the baseline system's performance is investigated.

\section{APPLICATION OF THE BASELINE SYSTEM}

In this section, a description of the nonlinear aircraft engine model is given. Based on this model, The LOBEM is developed, followed by the derivation of threshold values used by the FDI logic. For the results presented in this paper, the same nonlinear engine model used to generate the baseline system is used to represent a real engine.

\section{Engine Model}

The engine model used in this paper is a nonlinear simulation of an advanced high-bypass turbofan engine, a typical power plant for a large commercial aircraft. This engine model has been constructed as a component level model, which consists of the major components of an aircraft engine. The engine model captures highly complex engine physics. Engine performance deviations from the nominal health condition are modeled by adjustments to efficiency and flow capacity scalars of the following five components: fan (FAN), booster (BST), high-pressure compressor (HPC), high-pressure turbine (HPT), and low-pressure turbine (LPT). There are a total of 10 adjustments that are called health parameters. The engine state variables, health parameters, actuator variables, and environmental parameters are shown in Table 1.

There are a total of 10 measured parameters $(y$ and $z$ ) that are available to the digital control unit of this engine. Table 2 shows seven measured parameters $(y)$ along with their standard deviations given in percent of steady-state values at the ground maximum power condition. These parameters are measured by dual-channel sensors with channels A and B. It is assumed that both channels have the same statistical characteristics. The control action and diagnostics are based on those sensed variables. Table 3 shows three additional measured parameters (z) along with their standard deviations given in their actual engineering units. These three parameters indicate the ambient and engine inlet conditions. The two measured parameters $\mathrm{P}_{\mathrm{amb}}$ and P2 are used to calculate altitude and Mach number. The measurements of the inlet condition, T2 and P2, are used for parameter correction [8]. These three parameters are necessary to run the LOBEM over the flight envelope. Faults in these parameters are not considered in this paper.

The nonlinear engine model is used in the subsequent sections to represent a real engine in Eq. (1). The engine operates at given health conditions, and its flight condition is specified by the three environmental parameters listed in Table 1. This engine operates in closed loop with a control system

TABLE 1. ENGINE MODEL VARIABLES

\begin{tabular}{|c|c|}
\hline State Variables & $\begin{array}{l}\text { XNL, XNH, TMHS23, TMHS3 } \\
\text { TMHSBL, TMHSBC, TMHS41 } \\
\text { TMHS42, TMHS5 }\end{array}$ \\
\hline $\begin{array}{c}\text { Health } \\
\text { Parameters }\end{array}$ & $\begin{array}{l}\text { FAN efficiency, FAN flow capacity } \\
\text { BST efficiency, BST flow capacity } \\
\text { HPC efficiency, HPC flow capacity } \\
\text { HPT efficiency, HPT flow capacity } \\
\text { LPT efficiency, LPT flow capacity }\end{array}$ \\
\hline Actuators & WF36, VBV, VSV \\
\hline $\begin{array}{c}\text { Environmental } \\
\text { Parameters }\end{array}$ & $\begin{array}{l}\text { Altitude, Mach Number } \\
\text { Ambient Temperature }\end{array}$ \\
\hline
\end{tabular}

TABLE 2. STANDARD DEVIATIONS OF CONTROLS AND DIAGNOSTICS SENSORS ( $\sigma$ IN \% OF STEADY-STATE VALUES AT GROUND MAXIMUM POWER CONDITION)

\begin{tabular}{cc}
\hline \hline Sensors $(y)$ & $\sigma(\%)$ \\
\hline XN12 & 0.25 \\
XN25 & 0.25 \\
P25 & 0.50 \\
T25 & 0.75 \\
PS3 & 0.50 \\
T3 & 0.75 \\
T49 & 0.75 \\
\hline \hline
\end{tabular}

TABLE 3. STANDARD DEVIATIONS OF AMBIENT AND ENGINE INLET SENSORS ( $\sigma$ IN ACTUAL UNITS)

\begin{tabular}{cc}
\hline \hline Sensors $(\mathrm{z})$ & $\sigma$ \\
\hline $\mathrm{P}_{\mathrm{amb}}$ & $0.1 \mathrm{psi}$ \\
$\mathrm{T} 2$ & $5.0^{\circ} \mathrm{F}$ \\
$\mathrm{P} 2$ & $0.1 \mathrm{psi}$ \\
\hline
\end{tabular}


described in Ref. [9]. In the current control architecture, the power lever angle (PLA) is converted to desired corrected fan speed (an indicator of thrust). The control system adjusts actuation variables to cause the corrected measured fan speed to match the desired value. The closed-loop system runs at the frequency of $50 \mathrm{~Hz}$.

\section{Linear On-Board Engine Model}

The LOBEM is developed through the linearization of the nonlinear engine model in Eq. (3) at multiple operating points over the flight envelope. During this linearization process, the health condition of the nonlinear engine model is set to a specific condition (e.g., nominal health condition), and this condition is called the health baseline. The piecewise linear models are then saved in table lookup form. Figure 1 shows the structure of the LOBEM. The LOBEM is partitioned into two segments: steady-state segment (steady-state vectors) and dynamic segment (state-space matrices). The steady-state segment captures nonlinear engine characteristics along the engine's steady-state operating line, while the dynamic segment captures the engine's behavior due to perturbations from the steady-state operating line. These two segments are in table lookup form and interpolated based on scheduling parameters as the operating condition of the real engine moves from one point to another. The LOBEM generates the expected engine outputs at operating conditions over the flight envelope.

The number of scheduling parameters corresponds to the dimension of the LOBEM that must be interpolated. It is desirable to keep this dimension as small as possible so that the LOBEM can be run in real time with little computational burden. As mentioned in Ref. [10], it is essential to have an accurate representation of the expected steady-state engine performance characteristics. Also mentioned is that it is not necessary to schedule both segments with the same scheduling parameters. Based on this observation, the LOBEM's steadystate and dynamic segments are interpolated differently.

The steady-state segment is composed of steady-state vectors generated at operating conditions over the full range of altitude, Mach number, and PLA. The steady-state segment is interpolated by three scheduling parameters: computed altitude, computed Mach number, and computed corrected fan speed. The computed corrected fan speed is a state variable of the LOBEM and is closely related to power setting. As mentioned in the "Engine Model" section, altitude and Mach number are computed from P2 and $\mathrm{P}_{\mathrm{amb}}$ measurements. If altitude and Mach number measurements are available, the measured values can be used instead of computed values.

The dynamic segment is composed of state-space matrices generated at the sea-level-static and cruise operating conditions over the full range of PLA. The dynamic segment is interpolated by two scheduling parameters: $\mathrm{P} 2 / \mathrm{P}_{\mathrm{amb}}$ and computed corrected fan speed.

The steady-state segment is set up in three dimensions while the dynamic segment is set up in two. With this

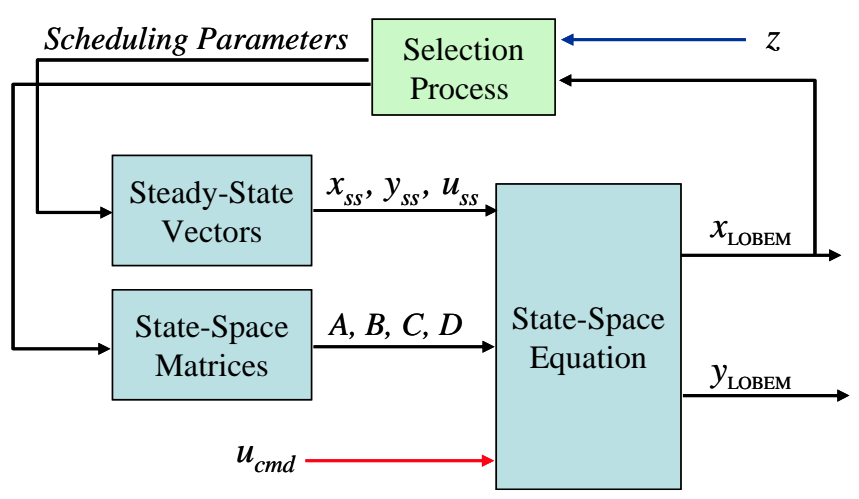

FIGURE 1. STRUCTURE OF THE LOBEM

structure, the LOBEM accurately represents the real engine's behavior over the flight envelope while keeping the computational burden low.

\section{Selection of Threshold Values}

In the diagnostic approach based on triplex channels, multiple thresholds must be determined. First, the threshold $\tau_{D R}^{i}$ for the dual-channel residual in Eq. (5) must be determined for each of the $m$ sensors. Similarly, the threshold $\tau_{A R}^{i}$ for the analytical residual in Eq. (6) must be determined for each of the $m$ sensors. The same threshold value is used for both channels of each sensor. Finally, the threshold $\tau_{\text {CFS }}$ for CFS in Eq. (7) must be determined for channels A and B, and the same threshold value is used for both channels. The total number of threshold values that must be determined is $2 m+1$.

The threshold values for dual-channel residuals can be determined relatively easily. The dual-channel residuals are neither influenced by engine health degradation nor component faults. They are influenced only by sensor failure and sensor noise. Thus, the main concern for determining the threshold values is to make sure that the sensor noise does not cause a threshold violation. Based on this consideration, the threshold values can be determined for seven dual-channel sensors in Table 2.

Unlike the dual-channel residuals, the analytical residuals are influenced not only by sensor failure and sensor noise but also by other factors such as engine health degradation, modelplant mismatch, and component faults. Similarly, CFS is influenced by the same factors except sensor failure. Since health degradation and model-plant mismatch are not considered to be faults, it is necessary to assure that these factors do not cause a threshold violation.

As discussed in an earlier section, the influence of engine health degradation must be accounted for by periodically updating the LOBEM to a new health baseline. As long as the health baseline of the LOBEM is maintained in the close 
vicinity of the degraded engine's true health condition, the analytical residuals and CFS can be maintained within a reasonable range of their nominal condition values.

The model-plant mismatch is another factor that must be considered in determining the threshold values. In the real environment, some degree of mismatch between the real engine and the LOBEM always exists due to unmodeled and incorrectly modeled physical phenomena. To make sure that the model-plant mismatch does not cause a threshold violation, the threshold values are determined through the same process taken in Refs. [9,11]. In this process, the influence of modelplant mismatch on analytical residuals and CFS is investigated through Monte-Carlo simulation; the nonlinear engine model (representing a real engine) and the LOBEM are run with different levels of health condition mismatch between them. The values of analytical residuals and CFS vary over a certain range because of this mismatch. The thresholds are set to values higher than the maximum values that the analytical residuals and CFS can reach due to mismatch. With this approach, it can be ensured that a threshold violation does not occur at least for the mismatch used in the simulated cases.

In the current setup, dual-channel residuals, analytical residuals, and CFS are processed by a low-pass filter with a cutoff frequency of $1.0 \mathrm{rad} / \mathrm{sec}(0.16 \mathrm{~Hz})$. All these signals are compared against the thresholds, and a threshold violation is declared when any signal persistently exceeds its corresponding threshold. For this study, a threshold violation is declared when a threshold is exceeded 25 consecutive time steps $(0.5 \mathrm{sec})$. This persistency test is carried out to ensure the existence of a fault. Based on the threshold violations occurring from the engine, the FDI logic determines the root cause of the anomaly. The low-pass filter design and persistency test are adjusted based on the engineering judgment of the designer. The performance of the diagnostic system will vary with those design factors.

\section{PERFORMANCE EVALUATION}

In this section, the performance of the baseline system is evaluated in a simulation environment using faults in sensors and components. Figure 2 shows the simulation setup. The nonlinear engine model is used to represent the real engine. Since accommodation of sensor faults is beyond the scope of this paper, the control system is configured to use the measurements on channel A regardless of fault existence. Sensor faults are injected into channels A and B individually to investigate the effect of control action.

The baseline system is evaluated at a cruise condition, and the threshold values at this operating point are shown in Table 4. ${ }^{\ddagger}$ The evaluation is conducted at the ideal condition; there is no seeded model-plant mismatch between the engine and the

\footnotetext{
${ }^{\ddagger}$ For full flight envelope evaluation, different threshold values should be used at different operating points, and the adaptive threshold approach discussed in Ref. [9] could be used during transient operation.
}

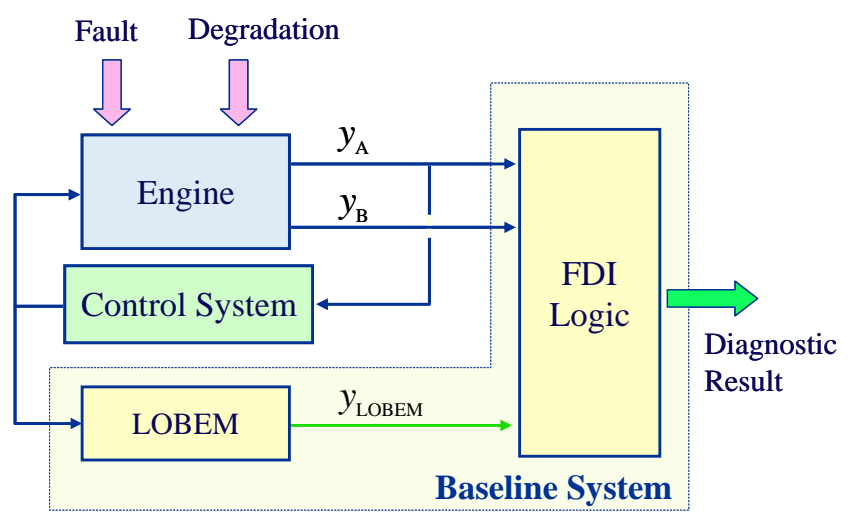

FIGURE 2. SIMULATION SETUP OF THE BASELINE SYSTEM

TABLE 4. THRESHOLD VALUES AT CRUISE CONDITION

\begin{tabular}{|c|c|c|}
\hline Sensors & $\tau_{D R}(\sigma)$ & $\tau_{A R}(\sigma)$ \\
\hline XN12 & \pm 2.0 & \pm 3.6 \\
\hline XN25 & \pm 2.0 & \pm 5.2 \\
\hline P25 & \pm 2.0 & \pm 1.5 \\
\hline $\mathrm{T} 25$ & \pm 2.0 & \pm 1.4 \\
\hline PS3 & \pm 2.0 & \pm 1.7 \\
\hline T3 & \pm 2.0 & \pm 1.6 \\
\hline $\mathrm{T} 49$ & \pm 2.0 & \pm 1.6 \\
\hline$\tau_{C F S}=1.2$ & & \\
\hline
\end{tabular}

LOBEM, other than the mismatch incurred by the linearization of the nonlinear engine model. Also, the same noise characteristics are used for both channels A and B. The robustness of the baseline system to engine health degradation is investigated first.

\section{Robustness to Engine Health Degradation}

The baseline system eventually diagnoses engine health degradation as a fault unless the health baseline (design health condition) of the LOBEM is updated to a new health condition. In this section, the level of health degradation at which the baseline system starts indicating the existence of a fault is determined. The engine is simulated to degrade gradually over its lifetime along the health degradation profile shown in Fig. 3. This degradation profile was developed in Ref. [7]. The life span of the engine is divided into 300 sample points in this example. The health condition at the $k^{\text {th }}$ sample point is defined by the vector $h_{k}$ in Eq. (9). At each sample point, the engine is run at a steady-state cruise condition with a given health condition. While the engine degrades over its lifetime, the 

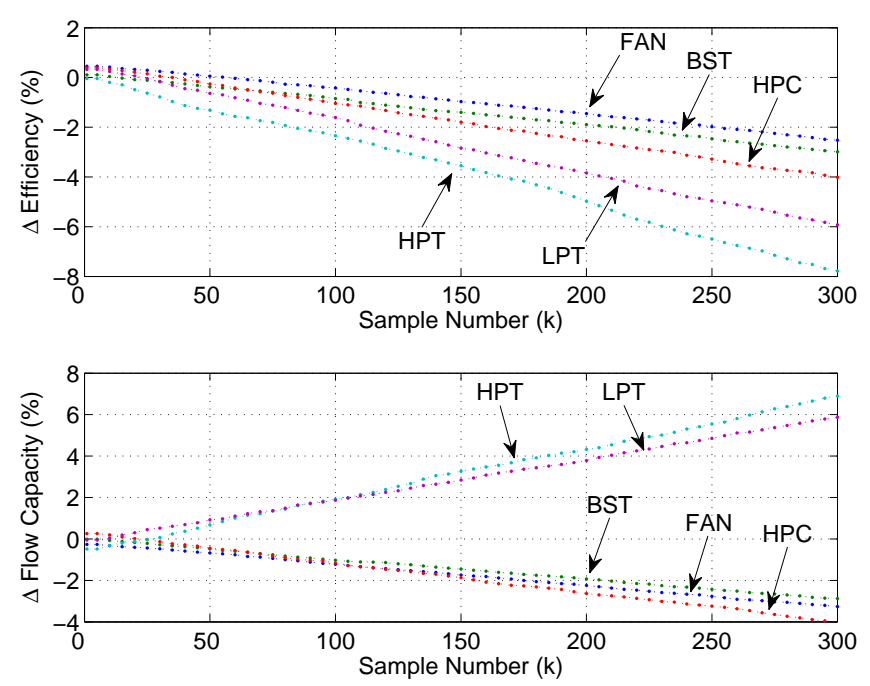

FIGURE 3. ENGINE HEALTH DEGRADATION PROFILE

health baseline of the LOBEM is maintained at the initial design condition: the nominal health.

The baseline system starts indicating the existence of a component fault at the $37^{\text {th }}$ sample point of the health degradation profile. At this point, only one health parameter has reached $1 \%$ deviation. This level of health degradation is far from the condition where a maintenance action is necessary. Thus, the diagnostic result, if trusted, can lead to an unnecessary maintenance action or inspection. One of the approaches to avoid such an undesirable scenario is to set the thresholds to much higher values so that a threshold violation does not occur until the health condition reaches a severe or full deterioration level. This approach, however, will compromise the diagnostic capability; the missed detection rate will increase. Another approach is to periodically update the LOBEM as discussed earlier. Although practical issues must be resolved, the model update is a necessary step to maintain the on-line diagnostic capability over the lifetime of an engine.

\section{Sensor Fault Diagnostics}

The baseline system's capability to detect and isolate a biased sensor is evaluated in this section. A bias is injected into channel A or B of a single sensor at a time. The health condition of the engine and the health baseline of the LOBEM are set to the nominal health. In the presence of a sensor bias, the closed-loop system is trimmed at a cruise operating condition. Then, the simulation in Fig. 2 is run at steady-state for 100 seconds. When any of the signals (dual-channel residuals, analytical residuals, and CFS) exceed a threshold for 25 consecutive time steps, a threshold violation is declared. Based on the threshold violations occurring from the engine, the FDI logic determines the root cause.
Table 5 shows the result of sensor fault diagnostics. The numbers in parenthesis are the results for cases where a bias is injected into channel B (open-loop configuration). The Anomaly Detection column shows the level of bias at which the baseline system detects the existence of an anomaly. At this point, the nature of the anomaly cannot be determined. The Sensor Fault Detection column shows the level of bias at which the baseline system detects a faulty dual-channel sensor. At this point, the identity of the faulty sensor is determined, but the identity of the faulty channel cannot be determined. The Sensor Fault Isolation column shows the level of bias at which the baseline system identifies the faulty channel of a faulty sensor. For instance, when a bias of $1.4 \sigma$ is injected into channel A of the P25 sensor, at least one of the analytical residuals exceeds a threshold, indicating the existence of an anomaly. When the bias is $1.8 \sigma$, the dual-channel residual of the P25 sensor exceeds its threshold, and therefore a fault is detected in this sensor. At the same bias magnitude, the analytical residual of this sensor also exceeds the threshold in channel $\mathrm{A}$, and the baseline system declares that channel $\mathrm{A}$ of the P25 sensor is faulty. When the XN12 or XN25 sensor is biased, the dual-channel residuals of these sensors exceed their threshold before any of the analytical residuals do. Therefore, anomaly detection does not occur for these two sensors.

The table shows that the sensor fault detection is achieved at the same bias level for all seven sensors. This is due to the fact that the thresholds for dual-channel residuals are set to the same value for all seven sensors, as shown in Table 4. It should be noted that the sensor fault detection, which is achieved through the comparison of dual-channel measurements of each sensor, is not influenced by control action, and thus the result is not affected whether a biased channel is A or B.

For those sensors other than XN12 and XN25, sensor fault

TABLE 5. SENSOR FAULT DIAGNOSTIC RESULT

\begin{tabular}{|c|c|c|c|}
\hline & $\begin{array}{c}\text { Anomaly } \\
\text { Detection }(\sigma)\end{array}$ & $\begin{array}{c}\text { Sensor Fault } \\
\text { Detection }(\sigma)\end{array}$ & $\begin{array}{l}\text { Sensor Fault } \\
\text { Isolation }(\sigma)\end{array}$ \\
\hline XN12 & --- / --- & $1.8 /-1.8$ & $\begin{array}{c}3.4 /-3.6 \\
(3.5 /-3.5)\end{array}$ \\
\hline XN25 & --- / --- & $1.8 /-1.8$ & $\begin{array}{c}2.9 /-4.2 \\
(5.0 /-5.0)\end{array}$ \\
\hline P25 & $\begin{array}{c}1.4 /-1.5 \\
(1.3 /-1.3)\end{array}$ & $1.8 /-1.8$ & $\begin{array}{c}1.8 /-1.8 \\
(1.8 /-1.8)\end{array}$ \\
\hline T25 & $\begin{array}{c}1.3 /-1.4 \\
(1.2 /-1.3)\end{array}$ & $1.8 /-1.8$ & $\begin{array}{c}1.8 /-1.8 \\
(1.8 /-1.8)\end{array}$ \\
\hline PS3 & $\begin{array}{c}1.5 /-1.6 \\
(1.5 /-1.6)\end{array}$ & $1.8 /-1.8$ & $\begin{array}{c}1.8 /-1.8 \\
(1.8 /-1.8)\end{array}$ \\
\hline T3 & $\begin{array}{c}1.4 /-1.6 \\
(1.4 /-1.5)\end{array}$ & $1.8 /-1.8$ & $\begin{array}{c}1.8 /-1.8 \\
(1.8 /-1.8)\end{array}$ \\
\hline T49 & $\begin{array}{c}1.4 /-1.5 \\
(1.4 /-1.5)\end{array}$ & $1.8 /-1.8$ & $\begin{array}{c}1.8 /-1.8 \\
(1.8 /-1.8)\end{array}$ \\
\hline
\end{tabular}

The numbers in parenthesis are the result for cases where a bias is injected into channel B (open-loop configuration). 
detection and isolation are achieved at the same bias level. This is a desirable result. When a sensor fault is detected, it is desirable to isolate the sensor problem to the specific faulty channel as early as possible. By knowing the root cause of the detected problem, the follow-up corrective actions can be taken sooner.

For the XN12 and XN25 sensors, sensor fault isolation is achieved at much higher bias levels than sensor fault detection. The difficulty in identifying the faulty channel for these two sensors is due to the threshold values for analytical residuals. As shown in Table 4, the thresholds for analytical residuals are set to much higher values for these two sensors than the other sensors. Higher threshold values are selected for fan and core speeds since they are highly coupled with control action, and the analytical residuals of these two sensors vary over a wide range in the presence of model-plant mismatch.

The influence of control action can be observed most prominently when the XN25 sensor is biased; the level of bias being isolated is much lower in channel A (closed-loop) than channel B (open-loop). This is because core speed (XN25) is the primary variable used in scheduling the VSV position. When a biased XN25 value is used by the control system, the engine is driven to a new steady-state condition. At the new operating condition, the relationship between the engine variables are "off-design" with respect to the original relationship at which the LOBEM was developed. This offdesign closed-loop effect further aggravates the output mismatch between the engine and the LOBEM and actually helps the baseline system to detect biases of smaller magnitude.

\section{Component Fault Detection}

The baseline system's capability to detect component faults is evaluated in this section. The health condition of the engine and the health baseline of the LOBEM are set to the nominal health. A component fault is represented by an abrupt shift in a health parameter, and it results in transient operation of the closed-loop system until the engine settles to new trim point. After the injection of a component fault, the engine is run for 100 seconds during which a diagnostic result is generated continuously by the baseline system. Because of the transient operation, the baseline system may indicate the existence of an anomaly for a certain period and then indicate the existence of a component fault. During this simulation run, if the baseline system indicates the existence of a component fault at least once, it is considered that the component fault is detected. If the baseline system does not indicate the existence of a component fault but indicates the existence of an anomaly at least once, it is considered that an anomaly is detected. If none of the above occurs (i.e., the baseline system indicates that a fault does not exist), it is considered that the baseline system misses the component fault.

Table 6 shows the component fault scenarios used in this evaluation study. The same scenarios were used as in Ref. [9]. Fault scenarios 1 through 5 represent single-component fault
TABLE 6. COMPONENT FAULT SCENARIOS

\begin{tabular}{cc}
\hline \hline Fault Scenario & Faulty Components \\
\hline 1 & FAN \\
2 & BST \\
3 & HPC \\
4 & HPT \\
5 & LPT \\
6 & FAN \& BST \\
7 & BST \& HPC \\
8 & FAN \&BST \& HPC \\
9 & HPT \& LPT \\
\hline & \\
Damage Level & Range of Fault Magnitude \\
\hline 1 & {$[1 \%, 2 \%]$} \\
3 & {$[2 \%, 3 \%]$} \\
4 & {$[4 \%, 4 \%]$} \\
\hline \hline
\end{tabular}

cases while fault scenarios 6 through 9 represent multiplecomponent fault cases. For each fault scenario, four levels of component damage are considered for evaluation. At each damage level, the efficiency and flow capacity of the faulty component(s) are independently shifted through a random process within the range shown in the table. This range is considered to encompass reasonable failure scenarios. All component shifts are made in the negative direction, except for HPT and LPT flow capacities which are shifted in the positive direction. At each damage level of each fault scenario, 100 fault cases are generated by randomly shifting health parameters. Thus, a total of 3600 component fault cases are used in the evaluation.

Table 7 shows the result of the component fault diagnostics. There are three possible diagnostic results that the baseline system may produce: 1) component fault detection, 2) anomaly detection, and 3) missed detection. In each cell of the table, the number of component fault cases that resulted in each of the three possible conditions appears in the aforementioned order. The best result that the baseline system can achieve is $100 / 0 / 0$. The result $0 / 100 / 0$ indicates that the baseline system detects the existence of an anomaly but cannot determine the nature of the anomaly. The result $0 / 0 / 100$ indicates that the baseline system completely misses the component faults.

It can be observed from the table that the baseline system is able to detect faults in HPC, HPT, and LPT. It has some difficulty in detecting BST faults when the fault level is 3 or below. The baseline system encounters the most difficulty in detecting FAN faults; it misses most of the FAN fault cases. As discussed in Ref. [9], this is due to the fact that the measurement shifts induced by FAN faults are difficult to observe through the sensor set used in this study.

It should be noted that the baseline system only indicates the existence of component faults without identifying the 
TABLE 7. COMPONENT FAULT DIAGNOSTIC RESULT

\begin{tabular}{cccccc}
\hline $\begin{array}{c}\text { Fault } \\
\text { Scenario \# }\end{array}$ & $\begin{array}{c}\text { Faulty } \\
\text { Components }\end{array}$ & Level 1 & Level 2 & Level 3 & Level 4 \\
\hline 1 & FAN & $0 / 0 / 100$ & $0 / 0 / 100$ & $5 / 3 / 92$ & $46 / 6 / 48$ \\
\hline 2 & BST & $0 / 0 / 100$ & $0 / 0 / 100$ & $20 / 27 / 53$ & $100 / 0 / 0$ \\
\hline 3 & HPC & $37 / 12 / 51$ & $100 / 0 / 0$ & $100 / 0 / 0$ & $100 / 0 / 0$ \\
\hline 4 & HPT & $100 / 0 / 0$ & $100 / 0 / 0$ & $100 / 0 / 0$ & $100 / 0 / 0$ \\
\hline 5 & LPT & $98 / 0 / 2$ & $100 / 0 / 0$ & $100 / 0 / 0$ & $100 / 0 / 0$ \\
\hline 6 & FAN/BST & $0 / 0 / 100$ & $0 / 0 / 100$ & $0 / 3 / 97$ & $99 / 0 / 1$ \\
\hline 7 & BST/HPC & $43 / 27 / 30$ & $100 / 0 / 0$ & $100 / 0 / 0$ & $100 / 0 / 0$ \\
\hline 8 & FAN/BST/HPC & $47 / 26 / 27$ & $100 / 0 / 0$ & $100 / 0 / 0$ & $100 / 0 / 0$ \\
\hline 9 & HPT/LPT & $100 / 0 / 0$ & $100 / 0 / 0$ & $100 / 0 / 0$ & $100 / 0 / 0$ \\
\hline \hline
\end{tabular}

Each cell shows the number of component fault cases that resulted in the following three categories:

Component fault detection, anomaly detection, and missed detection

specific faulty components. To determine the identity of faulty components, the measurements must be further processed through an algorithm, such as a bank of Kalman filters which was demonstrated in Ref. [12].

\section{Possible Design Adjustment}

The evaluation results presented in this section indicate the level of diagnostic performance that can be achieved at a cruise condition. If the performance is not satisfactory, the baseline system must be adjusted. The design elements that influence the achievable performance are 1) the sensor noise filter, 2) the persistency test, and 3) the threshold values. Among these three elements, a possible adjustment of the threshold values is specifically discussed in this section.

When heuristic knowledge regarding the target system is available, the threshold values should be adjusted accordingly. For instance, the evaluation results presented in this paper show that by applying a $2 \sigma$ threshold value for the dual-channel residuals (Table 4), a sensor fault is declared when the bias magnitude reaches $1.8 \sigma$ (Table 5 ). This means that any steadystate disagreement between the dual-channel measurements that exceeds $1.8 \sigma$ will be diagnosed as a sensor fault. In the real environment, disagreement between the channels may be induced by something other than a sensor fault. For instance, if the sensing elements of a dual-channel sensor are placed at different circumferential locations, the dual-channel measurements may not agree with each other because of three dimensional flow effects. If it is known that such a disagreement can be greater than the sensor fault detection level (1.8 $\sigma$ for the current design), the threshold should be set at a higher value in order to avoid false alarms.

Similarly, the thresholds for the analytical residuals can be adjusted in order to balance the anomaly detection capability and false alarm rates. This adjustment, however, should be done with care. If the threshold for an analytical residual is set much higher than the threshold for a dual-channel residual, the sensor fault isolation capability will suffer; it will take a much greater fault magnitude to be isolated than to be detected. This is the case for the XN12 and XN25 sensors. The objective of utilizing an analytical third channel is to isolate an in-range sensor fault. This objective is defeated if sensor fault isolation can only be achieved at a much greater fault magnitude than required for sensor fault detection to be achieved.

\section{DISCUSSION}

When the baseline system detects abnormal behavior of an aircraft engine, some actions must be taken to prevent undesirable engine operation. In this section, the corrective or accommodation actions that should be taken based on the baseline system's diagnostic results are discussed.

When the baseline system indicates the existence of an anomaly, the nature of that anomaly is unknown. In this case, an immediate action to accommodate the anomaly cannot be taken. Instead, the data generated by the baseline system must be further investigated. Anomaly detection is triggered when at least one of the analytical residuals exceeds a threshold while all dual-channel residuals and CFS remain below their threshold. These signals give some clue regarding the nature of the anomaly. For instance, when a sensor is faulty, the analytical residual of only one sensor should have exceeded its corresponding threshold while the analytical residuals of all other sensors and CFS remain at or near their nominal values. Moreover, the dual-channel residual of the same sensor should display some deviation from its nominal value, although it still remains below its corresponding threshold. On the other hand, when a component is faulty, some deviation from the nominal should appear in both channels of CFS and analytical residuals. As such, through the investigation of the data, the nature of the detected anomaly can be inferred.

When the baseline system detects a fault in a specific sensor, it is necessary to investigate the data generated by the baseline system and determine the failed channel. An 
immediate accommodation action can be taken only when the faulty channel of a faulty sensor is identified. If one channel is faulty, the measurement of the non-failed channel is used by the control system. If both channels of a particular sensor are faulty, the model output should be used.

Unlike the dual-channel sensors, the rotating components are not dual redundant. Therefore, the accommodation approach in the case of a component fault is fundamentally different from that of a sensor fault. When a component is faulty, it is important to know the engine's performance parameters such as thrust, compressor stall margins, and combustor temperature [13,14]. By knowing these parameters, the engine could be controlled within its operability limits while providing the thrust requested by the pilot. Thus, a reliable technique to estimate those un-measurable performance parameters is necessary for component fault accommodation.

A type of hardware failure, besides sensor and component faults, that was not discussed in this paper is an actuator fault. When an actuator fails to settle at a commanded position, this actuator is considered faulty. Generally, the actuator positions are measured, and the measured positions are fed back to the FADEC $[4,15]$. Detection and isolation of actuator faults can be achieved by comparing the commanded and measured actuator positions. When the difference exceeds a tolerance level for a specific actuator, this actuator is considered faulty. The actuators are not dual redundant, but different combinations of actuators can be used to achieve a certain objective. Therefore, an actuator fault could be accommodated through the manipulation of the remaining actuators based on pre-computed failure accommodation schedules [16] or adjustments [17].

\section{CONCLUSION}

The baseline system described in this paper was developed to diagnose aircraft gas turbine engine faults on-line (real-time, in-flight). The baseline system utilizes dual-channel sensor measurements and also the output of a linear on-board engine model (LOBEM) as the analytical third channel. Through the comparison of triplex channels, the baseline system diagnoses faults in sensors and rotating components.

The baseline system was evaluated extensively at a cruise operating condition using simulated fault cases. The baseline system exhibited its capability to identify a faulty dual-channel sensor and its failed channel at a reasonable fault level. The baseline system also exhibited its capability to detect component faults. To maintain such diagnostic capabilities, however, the baseline system must be updated periodically as the engine degrades over its lifetime. This periodic update is a major challenge that must be addressed for any on-line diagnostic algorithm which is designed at a specific health condition. The diagnostic capability of the baseline system establishes a benchmark for on-line diagnostics. Any improvement made through the application of advanced diagnostic techniques can be evaluated against this benchmark.

\section{REFERENCES}

[1] Rajagopalan, R., Wood, B. C., and Schryver, M., 2003, "Evolution of Propulsion Controls and Health Monitoring at Pratt and Whitney,” AIAA-2003-2645.

[2] Scoles, R. J., 1986, "FADEC - Every Jet Engine Should Have One,” SAE Paper 861802.

[3] Healy, T. A., Kerr, L. J., and Larkin, L. J., 1998, "Model Based Fuzzy Logic Sensor Fault Accommodation,” Journal of Engineering for Gas Turbines and Power, 120, pp. 533-536.

[4] Barclay, B. A., and Richards, J. C., 1977, "FADEC Preliminary Design Overview for Variable Cycle Engine Control,” AIAA Paper 77-837.

[5] Corley, R. C., and Spang, H. A., III, 1977, "Failure Detection and Correction for Turbofan Engines," Joint Automatic Control Conference, San Francisco.

[6] Merrill, W. C., DeLaat, J. C., and Bruton, W. M., 1998, "Advanced Detection, Isolation, and Accommodation of Sensor Failures - Real-Time Evaluation,” Journal of Guidance, Control, and Dynamics, 11, pp. 517-526.

[7] Kobayashi, T, and Simon, D. L., 2007, "Integration of OnLine and Off-Line Diagnostic Algorithms for Aircraft Engine Health Management,” ASME Paper GT2007-27518.

[8] Volponi, A. J., 1999, "Gas Turbine Parameter Corrections," Journal of Engineering for Gas Turbines and Power, 121, pp. 613-621.

[9] Kobayashi, T., and Simon, D. L., 2006, "Hybrid Kalman Filter: A New Approach for Aircraft Engine In-Flight Diagnostics,” NASA TM-2006-214491.

[10] Luppold, R. H., Roman, J. R., Gallops, G. W., and Kerr, L. J., 1989, "Estimating In-Flight Engine Performance Variations Using Kalman Filter Concepts,” AIAA-89-2584.

[11] Kobayashi, T., and Simon, D. L., 2006, "Hybrid Kalman Filter Approach for Aircraft Engine In-Flight Diagnostics: Sensor Fault Detection Case,” ASME Paper GT2006-90870.

[12] Volponi, A. J., DePold, H., Ganguli, R., and Chen, D., 2003, "The Use of Kalman Filter and Neural Network Methodologies in Gas Turbine Performance Diagnostics: A Comparative Study,” Journal of Engineering for Gas Turbines and Power, 125, pp 917-924. 
[13] Litt, J. S., 2005, “An Optimal Orthogonal Decomposition Method for Kalman Filter-Based Turbofan Engine Thrust Estimation,” ASME Paper GT2005-68808.

[14] Kobayashi, T., Simon, D. L., and Litt, J. S., 2005, "Application of a Constant Gain Extended Kalman Filter for In-Flight Estimation of Aircraft Engine Performance Parameters,” ASME Paper GT2005-68494.

[15] Barclay, B. A., 1976, "FADEC - Digital Propulsion Control of the Future,” AIAA Paper 76-652.
[16] Swan, J. A., and Vizzini, R. W., 1988, “Analytical Redundancy Design for Improved Engine Control Reliability, Final Review,” AIAA-88-3176.

[17] Rausch, R., Viassolo, D. E., Kumar, A., Goebel, K., Eklund, N., Brunell, B., and Bonanni, P., 2004, "Towards InFlight Detection and Accommodation of Faults in Aircraft Engines,” AIAA 2004-6463. 

APPENDIX

FLOW CHART OF THE FAULT DETECTION AND ISOLATION LOGIC

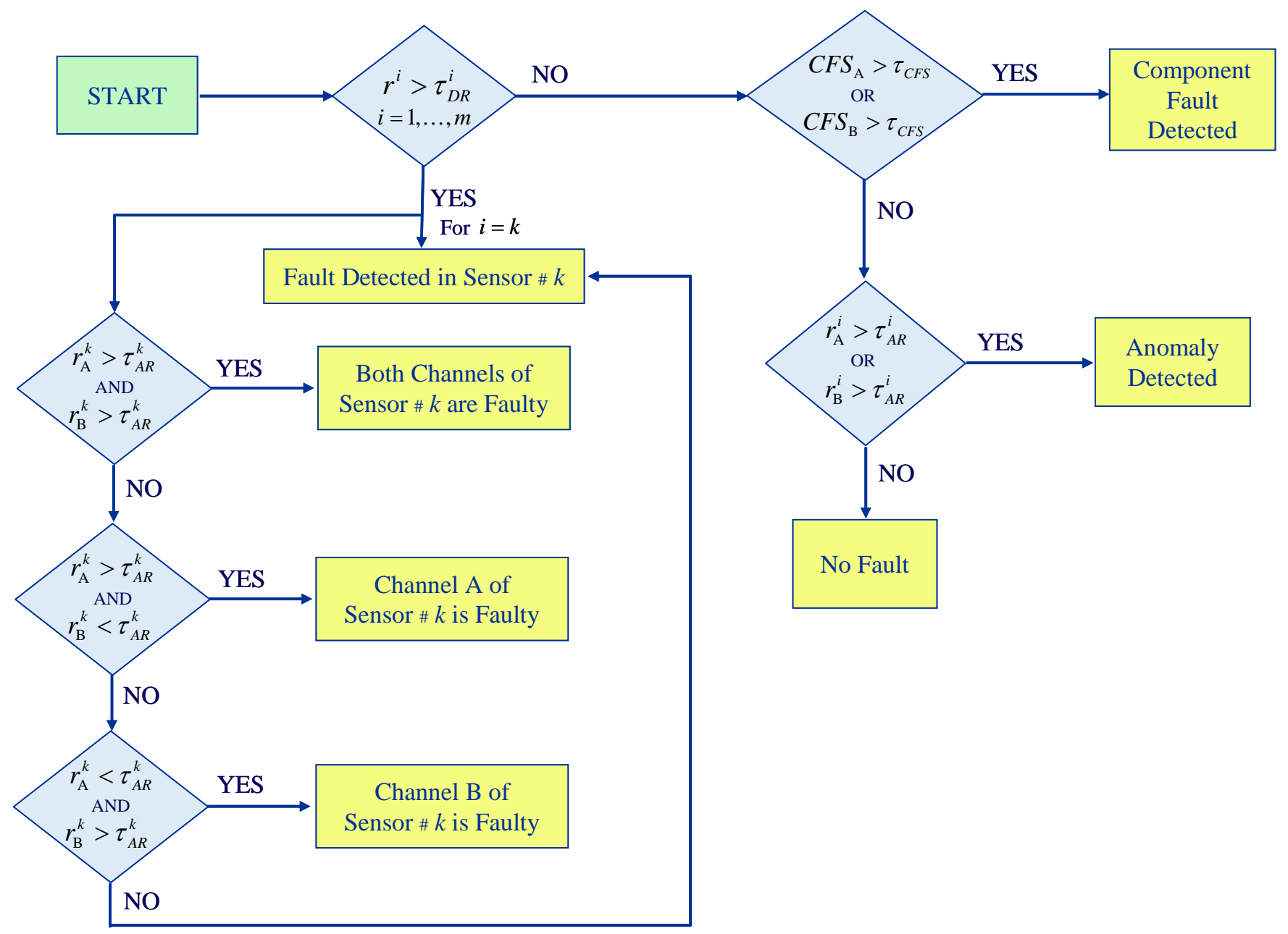




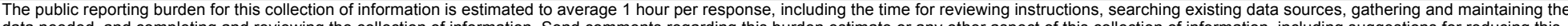

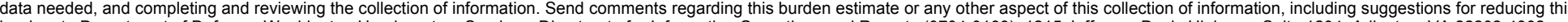

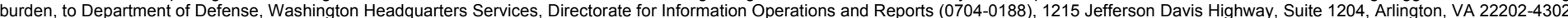

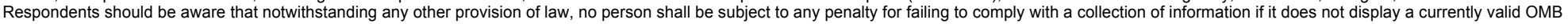
control number.

PLEASE DO NOT RETURN YOUR FORM TO THE ABOVE ADDRESS

\section{REPORT DATE $(D D-M M-Y Y Y Y)$ \\ 2. REPORT TYPE \\ 3. DATES COVERED (From - To)}

01-06-2008

Technical Memorandum

\section{TITLE AND SUBTITLE}

Aircraft Engine On-Line Diagnostics Through Dual-Channel Sensor Measurements:

Development of a Baseline System

\section{5a. CONTRACT NUMBER}

5b. GRANT NUMBER

5c. PROGRAM ELEMENT NUMBER

6. AUTHOR(S)

Kobayashi, Takahisa; Simon, Donald, L.

\section{5d. PROJECT NUMBER}

5e. TASK NUMBER

5f. WORK UNIT NUMBER

WBS 645846.02.07.03.03.01

8. PERFORMING ORGANIZATION REPORT NUMBER

E-16515

National Aeronautics and Space Administration

John H. Glenn Research Center at Lewis Field

Cleveland, Ohio 44135-3191

\section{SPONSORING/MONITORING AGENCY NAME(S) AND ADDRESS(ES)}

National Aeronautics and Space Administration

Washington, DC 20546-0001

\begin{tabular}{l}
$\begin{array}{l}\text { 10. SPONSORING/MONITORS } \\
\text { ACRONYM(S) } \\
\text { NASA }\end{array}$ \\
\hline $\begin{array}{l}\text { 11. SPONSORING/MONITORING } \\
\text { REPORT NUMBER } \\
\text { NASA/TM-2008-215228; GT2008-50345 }\end{array}$
\end{tabular}

\section{DISTRIBUTION/AVAILABILITY STATEMENT}

Unclassified-Unlimited

Subject Category: 07

Available electronically at http://gltrs.grc.nasa.gov

This publication is available from the NASA Center for AeroSpace Information, 301-621-0390

\section{SUPPLEMENTARY NOTES}

\section{ABSTRACT}

In this paper, a baseline system which utilizes dual-channel sensor measurements for aircraft engine on-line diagnostics is developed. This system is composed of a linear on-board engine model (LOBEM) and fault detection and isolation (FDI) logic. The LOBEM provides the analytical third channel against which the dual-channel measurements are compared. When the discrepancy among the triplex channels exceeds a tolerance level, the FDI logic determines the cause of the discrepancy. Through this approach, the baseline system achieves the following objectives: (1) anomaly detection, (2) component fault detection, and (3) sensor fault detection and isolation. The performance of the baseline system is evaluated in a simulation environment using faults in sensors and components.

\section{SUBJECT TERMS}

Aircraft engine diagnostics; Fault detection and isolation; On-board engine model; FADEC; On-line diagnostics; Dual-channel sensors;

\begin{tabular}{|c|c|c|c|c|}
\hline \multicolumn{3}{|c|}{ 16. SECURITY CLASSIFICATION OF: } & \multirow{2}{*}{$\begin{array}{l}\text { 17. LIMITATION OF } \\
\text { ABSTRACT } \\
\text { UU }\end{array}$} & \multirow{2}{*}{$\begin{array}{l}\text { 18. NUMBER } \\
\text { OF } \\
\text { PAGES } \\
20\end{array}$} \\
\hline $\begin{array}{l}\text { a. REPORT } \\
\text { U }\end{array}$ & $\begin{array}{l}\text { b. ABSTRACT } \\
U\end{array}$ & $\begin{array}{l}\text { c. THIS } \\
\text { PAGE } \\
\text { U }\end{array}$ & & \\
\hline
\end{tabular}
19a. NAME OF RESPONSIBLE PERSON STI Help Desk (email:help@sti.nasa.gov) 19b. TELEPHONE NUMBER (include area code) 301-621-0390



